https://helda.helsinki.fi

latrogenic patient injuries in otology during a 10-year period : review of national patient insurance charts

\title{
Helmiö, Paivi
}

2018

Helmiö , P , Saarinen , R , Aaltonen , L-M , Lehtonen , L \& Blomgren , K 2018 , ' latrogenic patient injuries in otology during a 10-year period : review of national patient insurance charts ' , Acta Oto-Laryngologica , vol. 138 , no. 1 , pp. 16-20 . https://doi.org/10.1080/00016489.2017.1375153

http://hdl.handle.net/10138/311683

https://doi.org/10.1080/00016489.2017.1375153

acceptedVersion

Downloaded from Helda, University of Helsinki institutional repository.

This is an electronic reprint of the original article.

This reprint may differ from the original in pagination and typographic detail.

Please cite the original version. 


\section{Patient Injuries in Otology During a Ten- Year Period: Review of National Patient Insurance Charts}

Päivi Helmiö, MD ${ }^{1}$, PhD, Leena-Maija Aaltonen, $\mathrm{MD}, \mathrm{PhD}^{2}$, Lasse Lehtonen, $\mathrm{MD}, \mathrm{PhD}^{3}$, Riitta Saarinen, $\mathrm{MD}, \mathrm{PhD}^{2}$, Karin Blomgren, $\mathrm{MD}, \mathrm{PhD}^{2}$

2 Department of Otorhinolaryngology, Helsinki University Hospital and University of Helsinki, Finland

${ }^{3}$ Department of Public Health, Helsinki University Hospital and University of Helsinki, Finland

${ }^{1}$ Department of Surgery, Turku University Hospital, Turku, Finland

\section{Corresponding author:}

Päivi Helmiö, MD, PhD

Turku University Hospital

Department of Surgery

P.O. Box 52

FI-20521 Turku

Finland

Tel: +358 503824667

Fax: +358 23131709

E-mail: pamais@utu.fi

Short title: Patient injuries in otology 


\section{Abstract}

Objectives: To assess patient injury characteristics and contributing factors in otology.

Study design: A retrospective claim record study of national patient insurance charts in Finland during a ten-year period.

Material and methods: Data of the accepted patient injury claims involving otorhinolaryngology (ORL), closed between the years 2001 and 2011, were obtained from a search of the Finnish Patient Insurance Centre registry. Injuries that concerned otology were included in this study. The cause and the type of injury were evaluated and classified.

Results: During the 10-year study period, altogether 44 claims were accepted as compensated patient injuries in otology. This was $18.9 \%$ from a total of 233 patient injuries for all ORL. Twelve (26.0\%) injuries occurred in outpatient care and 32 (72.7\%) injuries in surgical procedures. Five (11.4\%) patients were children. Errors in surgical technique were identified as the primary cause of injury in 22 (68.8\%) operation related cases. Mistakes with removable auricular tampons left in situ were contributing factors in $4(12.5 \%)$ injuries. Facial nerve was damaged in 9 (28.1\%) operations and the injuries resulted in the severe hearing loss or deafness in 12 (37.5\%) patients. Six patients (21\%) needed one or more reoperations because of the injury, out of which 2 due to incomplete primary operation.

Conclusions: Patient injuries in operative otology were caused by typical complications of common operations. 


\section{Introduction}

Patient safety is one of the most important goals for health care organizations. Prevention of errors and adverse events (AE) should be high priority within every specialty.

The patient's pathway through treatment process has to be analysed on a systemic basis for patient safety purposes ${ }^{1}$. The objective is to identify the root causes of AEs. Traditionally, specialties have taken the responsibility for the research of treatment protocols and operation techniques within their own domain. Results and complications have been analysed on a clinical basis that is related to the diseases and the surgical methods used. Not so much attention has been paid to the systemic causes of errors. Malpractice claims and insurance records constitute an important source of information on factors contributing to patient injury 2 .

A wide range of errors with technical, interactive or administrative cause constitutes a potential source of $\mathrm{AEs}{ }^{3}$. An $\mathrm{AE}$ in health care is generally defined as an unintended injury or complication that results in prolonged hospital stay, disability at the time of discharge or death, caused by healthcare management rather than the underlying disease itself 4,5 . The consequences of the AEs for the patient vary from a harmless inconvenience to permanent disability or even death ${ }^{6}$. Consequently, AEs can cause burden the health care providers and incur disproportionately high costs for the health care system.

Despite the importance of the topic, research of errors and patient safety in otology subspecialty, is limited to few studies. Previous information about malpractice claim data for otology originates from USja UK ${ }^{7-9}$. A study by Mathew et al. reported that in UK $26 \%$ of the claims in ORL between 1995 and 2010 concerned otology, and two-thirds (64.9\%) of the reported errors were related to surgical management ${ }^{8}$.

\section{Objectives}

The aims of present study were to determine the sources of error and the characteristics of patient injuries arising from otological practice. 


\section{Material and Methods}

In Finland, all health care providers have patient insurance according to the Patient Injuries Act (Potilasvahinkolaki 585/1986). Thus, patients treated by the official health care providers and practitioners are insured by the Finnish Patient Insurance Centre (PIC) ${ }^{10}$. Accepted and compensated claims are defined as patient injuries. A treatment injury is the most typical compensable injury. A treatment injury is a bodily injury caused by an examination, treatment or other similar action performed on the patient, or the failure to do so. Consequently, a treatment injury may be, for example, a post-operative complication, such as a nerve injury or a delay in diagnosis, which could have been avoided by an experienced professional.

All accepted patient injury claims within the ORL specialty which were closed between $1^{\text {st }}$ of November 2001 and 31 th of October 2011, were sought from PIC registry. The analysis of the data and the relationship of the injuries with WHO Surgical Checklist has been previously published ${ }^{11}$. Patient injuries concerning solely otology were included in the present study.

Age, gender, diagnosis and major co-morbidities of the patient in addition to information of health care providers and institutions were recorded as background data. All medical records, experts' assessments and compensation decisions of the included claims were reviewed. Two ORL specialists evaluated the operation-related injuries in detail.

Incidents and errors contributing to the injury were identified and classified. One or two noteworthy independent incidents were defined for each patient. The structure of the classification used was based on the care-flow-process of the patient. The original idea of this sort of classification for ORL was presented by Shah et al. ${ }^{12}$. The classification used in present study is modified by the authors and presented in Table 3.

Statistical analyses

For statistical processing, descriptive data were summarised using numbers and proportions (\%). Statistical analyses were carried out with IBM SPSS software version 23.0 for Mac. 
Ethical considerations

This was a retrospective claim record study. The study protocol and data search were approved by PIC. All information regarding the identities of patients and health- care providers was excluded from the data.

\section{Results}

During the ten-year study period, PIC accepted and compensated a total of 223 claims as patient injuries within ORL-specialty. The claims covered treatment given between years 1998 and 2011. 44 (19.7\%) of these injuries concerned otology and are included in the present study. Annual prevalence was 4.4 injuries per a year and a majority (32 (72.7\%)) of injuries were associated with operative care and the rest (12 (27.3\%)) resulted from an error in outpatient care. Annual amount of injuries are presented in Figure 1.

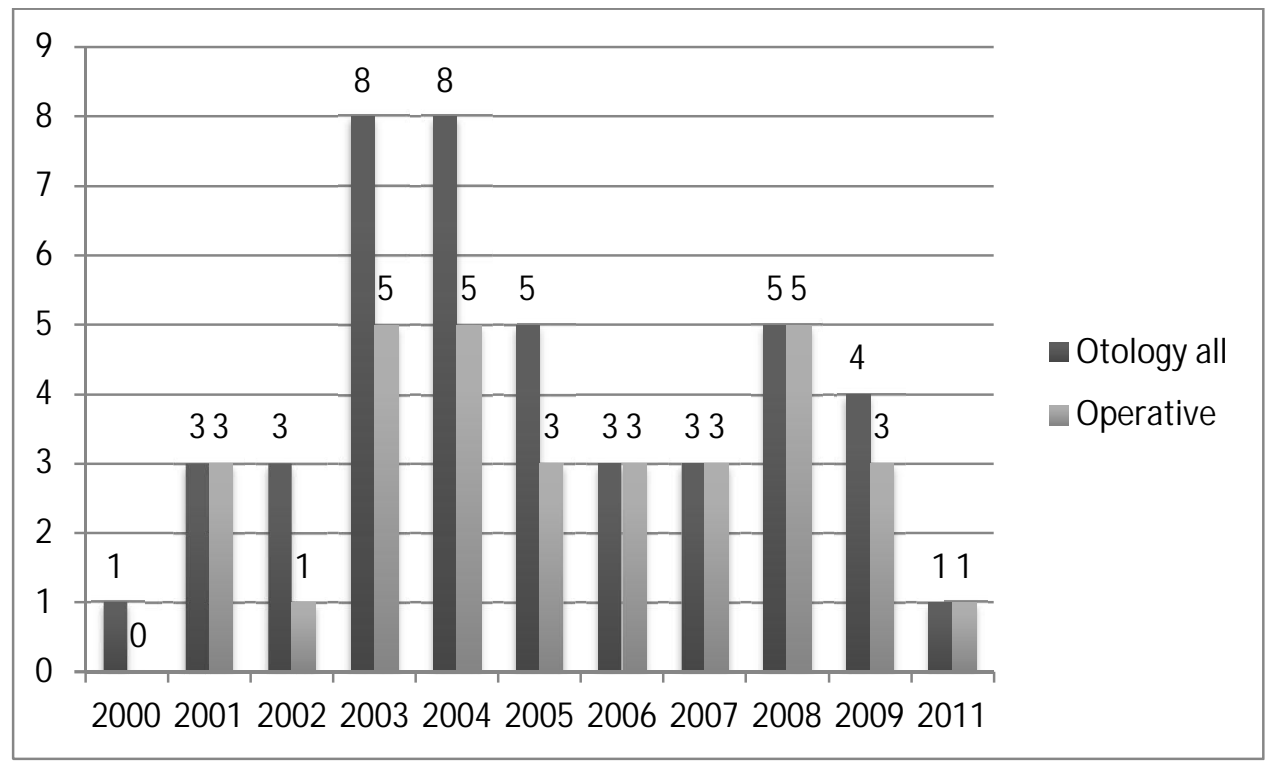

Figure 1.

Annual numbers (N) of accepted patient injury claims in otology closed between $1^{\text {st }}$ of November 2001 and $31^{\text {th }}$ of October 2011. N total $($ Otology all $)=44, \mathrm{~N}$ total $($ Operative $)=32$.

The mean age of the patients was 46 years (range 2-80 years) with 26 (59.1\%) being female. Five (11.9\%) patients were children under 16 year. The incidents and errors are presented in 
Table 1. In 19 cases (45.2\%), two noteworthy independent incidents, such as iatrogenic trauma to the facial nerve and postoperative infection due to retained packing after ear surgery for the same patient, were detected.

Table 1. Errors and incidents identified in otology related patient injuries during a ten-year study period, classified on the care flow basis.

\begin{tabular}{|c|c|c|c|c|}
\hline & \multicolumn{2}{|c|}{ Error or incident 1.} & \multicolumn{2}{|c|}{ Error or incident 2.* } \\
\hline & $\mathrm{N}$ & $\%$ & N & $\%$ \\
\hline \multicolumn{5}{|l|}{ OUTPATIENT CARE } \\
\hline Delay or error in diagnosis or treatment & 3 & 6.8 & 1 & 2.3 \\
\hline Injury in outpatient procedures & 8 & 18.2 & 0 & \\
\hline Other error in outpatient care & 1 & 2.3 & 0 & \\
\hline Outpatient care, all & 12 & 27.3 & 1 & 23 \\
\hline \multicolumn{5}{|l|}{ OPERATIVECARE } \\
\hline \multicolumn{5}{|l|}{ Preoperative judgement and surgical planning } \\
\hline Incorrect/unnecessary procedure or technique & 0 & & 0 & \\
\hline Insufficient patient information & 0 & & 1 & 2.3 \\
\hline Error in preoperative care & 0 & & 1 & 2.3 \\
\hline \multicolumn{5}{|l|}{ Operative unit } \\
\hline No prophylactic antibiotic & 1 & 2.3 & 0 & \\
\hline Problems in anaesthesia & 0 & & 0 & \\
\hline Wrong site surgery & 1 & 2.3 & 0 & \\
\hline Nerve lesion & 9 & 20.5 & 1 & 2.3 \\
\hline Other injury to adjacent anatomical structure & 5 & 11.4 & 0 & \\
\hline Incomplete surgery & 3 & 6.8 & 0 & \\
\hline Other errors in surgical technique & 5 & 11.4 & 0 & \\
\hline Haemostatic problem & 0 & & 0 & \\
\hline Retained gauze/instrument & 0 & & 0 & \\
\hline Equipment related errors & 0 & & 0 & \\
\hline Insufficient charts or instructions & 0 & & 3 & 6.8 \\
\hline Other error in operating theatre & 0 & & 0 & \\
\hline \multicolumn{5}{|l|}{ Postoperative period } \\
\hline Postoperative ward care & 0 & & 0 & \\
\hline Wrong/insufficient medication & 0 & & 0 & \\
\hline Infection & 3 & 6.8 & 2 & 4.5 \\
\hline
\end{tabular}




\begin{tabular}{|lllll|} 
Haemorrhage & 0 & & 0 & \\
Insufficient postoperative treatment/ follow up & 1 & 2.3 & 1 & 2.3 \\
Retained foreign body e.g. auricular tampons & 3 & 6.8 & 1 & 2.3 \\
Unintended outcome & 1 & 2.3 & 8 & 18.2 \\
Operativecare, all & $\mathbf{3 2}$ & $\mathbf{7 2 . 7}$ & $\mathbf{1 8}$ & $\mathbf{4 2 . 9}$ \\
Total & & & & \\
\hline
\end{tabular}

Number $(\mathrm{N})$ and proportion (\%) of errors detected in 44 accepted otology related patient injury claims. *In 19 (43.2\%) claims two independent errors contributing to the patient injury could be identified.

Injuries in Outpatient Care

Twelve (27.3\%) injuries resulted from an error in outpatient care and in one case it was a cofactor to the injury. Eight (18.2\%) injuries were due to the outpatient procedures. In six cases the injury occurred during wax removal from the external canal and in two cases when fitting for hearing aid. Otolaryngologist performed the procedure in one case, and in rest of the cases the performer was a nurse, a general practitioner or a medical student. In one case the general practitioner cleaning the ear canal was under the influence of alcohol. The errors resulted in inner ear damage, permanent tinnitus, perforation of the tympanic membrane and lesion to the ear canal. One patient fell and hit her head during vestibular rehabilitation. There were no cases of ototoxicity from topical medications in this data.

There were three (6.8\%) accepted claims for morbidity due to delayed treatment. In one case from year 2002 a child's hearing loss was missed resulting in a delay in cochlear implantation and speech development and in one case a delay in vestibular schwannoma surgery was noted. There was also a case of delay in referring a patient with sudden hearing loss and vertigo to a tertiary center causing a patient injury. In addition to these, delayed surgery was considered as a co-factor for facial nerve injury after cholesteatoma surgery.

\section{Surgical injuries}

Altogether 32 (72.7\%) of patient injuries were associated with operative care. A fully trained otolaryngologist performed thirty $93.8 \%$ operations and only in 2 (6.3\%) cases operator was a trainee. 27 (84.4\%) of the operations were performed in high volume university or central 
hospitals. Numbers of the units are presented in Table 2. All operations were elective and performed during office hours. Four (12.5\%) of the operated patients were children.

Table 2. Characteristics of health care providers and surgeons in operative otology patient injuries in Finland between 2001 and 2011.

\begin{tabular}{|lll|}
\hline & N & $\%$ \\
\hline Hospital & 12 & 37.5 \\
University hospital & 15 & 46.9 \\
Central hospital & 4 & 12.5 \\
Local hospital & 0 & 0 \\
Primary health care & 1 & 3.1 \\
Private health care provider & & \\
& & \\
Trainingof physician & 30 & 93.8 \\
ORL specialist & 2 & 6.3 \\
ORL trainee & 0 & 0 \\
Other & & \\
& 32 & 100 \\
Total & & \\
\hline
\end{tabular}

Number (N) and proportion (\%).

Patients were operated for common otological diseases. Twenty-three patients (71.9\%) were treated for diseases of the middle ear, 8 (25.0\%) for the external ear and one (3.1\%) patient underwent petrosectomy for the tumour of temporal glomus. Diagnoses that indicated operations are presented in Table 3. Mastoid surgery for the chronic middle ear infection or cholesteatoma was the most commonly associated with a patient injury, for 11 (34.4\%) cases. Otosclerosis surgery was the second most common cause of the injury. There were no patient injuries due to ventilation tube insertion and none of the patients was operated because of malignant disease.

Table 3. Operative diagnosis for the patients in operative otology related patient injuries.

\begin{tabular}{|llll|}
\hline & ICD-10 code & $N$ & $\%$ \\
\hline Extemal ear & & 1 & 3.1 \\
Atheroma of external ear & L72.1 & 1 & 3.1 \\
Otapostasis & Q17.5 & & \\
\hline
\end{tabular}




\begin{tabular}{|c|c|c|c|}
\hline Preauricular fistula & Q18.1 & 1 & 3.1 \\
\hline KK eksostoosi & $\mathrm{H} 61.8$ & 4 & 12.5 \\
\hline Chronic external ear infection & H66.3 & 1 & 3.1 \\
\hline \multicolumn{4}{|l|}{ Middle ear } \\
\hline Chronic middle ear infection & $\mathrm{H} 70.1$ & 5 & 15.6 \\
\hline Choleasteatoma & $\mathrm{H} 71$ & 6 & 18.8 \\
\hline Perforation of tympanic membrane & $\mathrm{H} 72.0$ & 1 & 3.1 \\
\hline $\begin{array}{l}\text { Perforation of tympanic membrane and damage to } \\
\text { ossicular chain }\end{array}$ & $\mathrm{H} 74.3$ & 1 & 3.1 \\
\hline Otosclerosis & $\mathrm{H} 80.0$ & 8 & 25.0 \\
\hline Conductive hearing loss, not otosclerosis. & $\mathrm{H} 90.2$ & 1 & 3.1 \\
\hline Congenital ossicular bone malformation & Q16.3 & 1 & 3.1 \\
\hline \multicolumn{4}{|l|}{ Intracranial } \\
\hline Tumor glomi temporale & D35.59 & 1 & 3.1 \\
\hline Total & & 32 & 100 \\
\hline
\end{tabular}

Number $(\mathrm{N})$ and proportion (\%) of operative diagnosis in 32 accepted operative otology related patient injury claims. ICD-10 =

Errors in surgical technique could be identified as the primary incident in 22 (68.8\%) cases. Incidental injury to a facial nerve occurred in 9 operations and injury to adjacent anatomical structures in 5 operations. One patient who underwent surgery for otosclerosis ended up with damage to inner ear and lesion of facial nerve. In two cases of exostosis surgery anterior wall of the ear canal collapsed due to extensive drilling causing a temporomandibular joint protrusion to the ear canal. There were 3 cases of incomplete surgery. In one case a preauricular fistula was operated three times due to incomplete surgery in the first two attemps. In one case of otapostasis the surgeon did not master the technique and the shape of the ear was not adequately corrected. In one case inner ear damage was caused by drilling the incompletely mobilized ossicular chain.

One wrong side operation was performed in this series. An experienced otolaryngologist in a university hospital started a tympanoplasty by endaural incision and liberated and turned the cartilagous part of the ear canal before the mistake was discovered. In that case multiple 
errors and confounding factors were identified: Hair was removed from the wrong side due to a misunderstanding arising from language problems and thus, a wrong side was prepared for the operation,

Mistakes with removable auricular tampons left in situ were contributing 4 (12.5\%) injuries. Facial nerve damage during the operation was the main reason for injury in one patient. The retained foreign material-related problems resulted in prolonged infections at the operation area in all 4 patients. In all cases the removal of aural tamponade at follow-up visit had been inadequate and the operation record charting of materials left in situ was insufficient or lacking. The time interval of foreign material removal varied from 3 weeks to 14 weeks.

No major systemic complications or deaths occurred in this material. The consequences of the injuries for the patient varied from extra trouble and unscheduled hospital visits to permanent disability (Table 4). Facial nerve was damaged in 9 (28.1\%) operations given rise to permanent morbidity in most cases. The consequence of injury was severe hearing loss or deafness in 12 (37.5\%) patients. In 6 (21\%) patients the injury resulted in one or more reoperations, out of which 2 were due to incomplete primary operation.

Table 4. The consequences of the operative injuries for the patient.

\begin{tabular}{|lll|}
\hline & $\mathrm{N}$ & $\%$ \\
\hline Facial nerve lesion & 7 & 21.9 \\
Facial nerve lesion and infection & 1 & 3.1 \\
Vertigo & 1 & 3.1 \\
Infection & 2 & 6.3 \\
Hearing loss & 8 & 25.0 \\
Hearing loss and facial nerve lesion & 1 & 3.1 \\
Hearing loss and vertigo & 2 & 6.3 \\
Hearing loss and tinnitus & 1 & 3.1 \\
Re-operation due to incomplete primary surgery & 2 & \\
Perforation of tympanic membrane & 1 & 3.1 \\
Other & 6 & \\
Total & & \\
\hline
\end{tabular}

Number (N) and proportion (\%) 


\section{Discussion}

Synopsis of key findings

Patient injuries in otology were strongly related to operative care and most of them were wellknown complications of otological procedures. Technical error in performing surgery was identified in two thirds of operation-related injuries.

Strengths and weaknesses of the study

Malpractice claim data and insurance records constitute a detailed source of information on injuries and their error sequences ${ }^{13}$. Our data was obtained from PIC insurance chart registry, which is nationwide and thus highly representative. However, with retrospectively collected data, we are dependent on information recorded in charts.

The PIC insurance compensates bodily damages to patients on a non- fault basis. A previous study has demonstrated that in Finland altogether $26.1 \%$ of claims for ORL have been accepted and compensated as patient injuries., The mean sum of compensation payment was $3320 € 14$ which is quite modest when compared to in the UK and US where the average indemnity sums have been $62700 £$ and 194924 \$7, . Although all patients in Finland are insured by PIC, it is likely that not all who had sustained an injury have submitted a claim. Thus, the overall incidence of injuries is presumably greater.

Comparisons with other studies

About $20 \%$ of the patient injuries and malpractice cases of ORL are related to the otology. Malpractice data from the US indicate that ear surgery comprised 18 (9\%) out of 200 claims related to operative ORL ${ }^{9}$. Correspondingly, $26 \%$ of the clinical negligence claims for ORL in the UK between 1995 and 2010, concerned otology, and out of these 63 (64.9\%) were due to operative complications ${ }^{8}$. In our series the respective rates were $19.7 \%$ and $72.7 \%$. Our study confirms that adverse events in otology are mostly associated with operative care.

Eight (18.2\%) injuries were due to the error in outpatient procedures; out of these six (13.6\%) were injuries after removal of wax from the external ear. Respective rates from the UK were 9.3\% and $7.2 \%$ 8. In Finland, nurses or general practitioners in primary health care services 
mostly perform this procedure, so, comparing the high volume of these procedures, injury rates can be considered low.

In UK, $15.5 \%$ of successful claims concerned of misdiagnosis/ delayed diagnosis and (4.1\%) of delayed surgery ${ }^{8}$. In our material, a delay in treatment or surgery was noted altogether in four (9.1\%) injuries. A delay in cochlear implantation happened in 2002. After that substantal changes in newborn's hearing screening have been made and emissions are nowadays checked from every newborn.

Patients from all age groups are represented in otology; in this series five (11.4\%) patients were children. Despite the high volume of ventilation tube insertion, there were no patient injuries due to myringotomies or tube insertions. During the early years of study period, adenotomy was still indicated for recurrent middle ear infection in children. These operations were classified as diseases of the adenoids and were excluded from this study. Mastoid surgery was the most common otologic surgery with an accepted claim, which is in correspondence with the previous studies ${ }^{8}$. Despite the possible devastating complications of advanced otological surgery, death and severe intracranial or systemic complications did not occur in our data.

A claim record study covering all surgical specialities noted that iatrogenic injury to adjacent structures was the most common cause for operation related injuries ${ }^{13}$. The auricular and temporal region includes several vulnerable anatomical structures that place high demands to surgical knowledge, techniques, and skills. The most commonly damaged structures are the facial nerve and the inner ear ${ }^{15,16}$ resulting, as expected, to hearing loss and facial paralysis. They were the the most common consequences of injury also in the present study. In the 18 ear surgery malpractice claims from the US the consequence was hearing loss in 10 (55.6\%) cases and facial nerve paralysis in seven (38.9\%) cases ${ }^{9}$. Conversely, $23 \%$ of the litigations for the iatrogenic facial nerve paralysis were due to otological operations and $40 \%$ of the head and neck malpractice cases were centred on damage to a cranial nerve ${ }^{9,15}$.

A fully trained otolaryngologist performed nearly all operations and a majority was performed in high volume hospitals. This is in accordance with results exploring US surgical claims from several surgical specialities ${ }^{13}$. Thus, traditionally recommended interventions 
like strict supervision of residents and restricting operations to high-volume hospitals, cannot address these errors. On the other hand, the number of mastoid surgery due to chronic middle ear infections, has in several centres, diminished.

A significant proportion of patient injuries in otology, e.g. wrong site surgery (WSS) and problems with removable packing left in situ, represent areas where safety should be addressed. Anatomical structures are bilateral and form a risk for wrong side surgery. A recent review by Liou et al. (2014) concluded that WSS accounts for 4-6\% of errors in ORL ${ }^{17}$ Six cases (9.5\%) of WSS were included in the 63 clinical negligence claims in operative otology in UK ${ }^{8}$. Our study includes one case of wrong side ear surgery, which explained 3.1\% of operationrelated injuries. WSS is obviously preventable, and interventions should be of high priority. Regardless of this, site-marking protocols have been variable and insufficient within the specialty 18,19 . Also, documentation of materials left in situ should always be structurally made. Fortunately, after the study period, implementation of surgical checklists has increased awareness of these important safety related issues and raised site marking as routine $2,11,20$.

Clinical applicability of the study

Knowledge and awareness of potential errors in treatment as well as understanding of the patterns underlying patient injuries is essential to develop preventive strategies. This study provides clinically useful information on errors in otology.

Conclusion

Patient injuries in otology are strongly related to operative care. Most injuries are well-known complications of procedures and occur in routine practice by fully trained ORL specialists.

\section{Conflict of interest}

None declared.

\section{Acknowledgements}

The Helsinki University Hospital Research Fund financially supported the study 


\section{References}

1 Roberson D.W., Kentala E. \& Healy G.B. (2004) Quality and safety in a complex world: why systems science matters to otolaryngologists. Laryngoscope. 114, 1810-1814

2 de Vries E.N., Eikens-Jansen M.P., Hamersma A.M. et al. (2011) Prevention of surgical malpractice claims by use of a surgical safety checklist. Ann Surg. 253, 624-628

3 Andrews L.B., Stocking C., Krizek T. et al. (1997) An alternative strategy for studying adverse events in medical care. Lancet. 349, 309-313

4 Thomas E.J., Studdert D.M., Burstin H.R. et al. (2000) Incidence and types of adverse events and negligent care in Utah and Colorado. Medical care. 38, 261-271

5 Bosma E., Veen E.J. \& Roukema J.A. (2011) Incidence, nature and impact of error in surgery. Br J Surg. 98, 1654-1659

6 Brennan T.A., Leape L.L., Laird N.M. et al. (1991) Incidence of Adverse Events and Negligence in Hospitalized-Patients - Results of the Harvard Medical-Practice Study-I. New Engl J Med. 324, 370-376

7 Dawson D.E. \& Kraus E.M. (2007) Medical malpractice and rhinology. American journal of rhinology. 21, 584-590

8 Mathew R., Asimacopoulos E. \& Valentine P. (2011) Toward safer practice in otology: a report on 15 years of clinical negligence claims. Laryngoscope. 121, 2214-2219

9 Hong S.S., Yheulon C.G., Wirtz E.D. et al. (2013) Otolaryngology and medical malpractice: A review of the past decade, 2001-2011. Laryngoscope

10 Finnish Patient Insurance Centre. Finnish Patient Insurance Centre

11 Helmio P., Blomgren K., Lehtivuori T. et al. (2015) Towards better patient safety in otolaryngology: characteristics of patient injuries and their relationship with items on the WHO Surgical Safety Checklist. Clin Otolaryngol. 40, 443-448

12 Shah R.K., Kentala E., Healy G.B. et al. (2004) Classification and consequences of errors in otolaryngology. Laryngoscope. 114, 1322-1335

13 Regenbogen S.E., Greenberg C.C., Studdert D.M. et al. (2007) Patterns of technical error among surgical malpractice claims: an analysis of strategies to prevent injury to surgical patients. Ann Surg. 246, 705-711

14 Lehtivuori T., Palonen R., Mussalo-Rauhamaa H. et al. (2013) Otorhinolaryngological patient injuries in Finland. Laryngoscope. 123, 2397-2400

15 Lydiatt D.D. (2003) Medical malpractice and facial nerve paralysis. Arch Otolaryngol Head Neck Surg. 129, 50-53

16 Svider P.F., Sunaryo P.L., Keeley B.R. et al. (2013) Characterizing liability for cranial nerve injuries: a detailed analysis of 209 malpractice trials. Laryngoscope. 123, 1156-1162

17 Liou T.N. \& Nussenbaum B. (2014) Wrong site surgery in otolaryngology-head and neck surgery. Laryngoscope. 124, 104-109

18 Shah R.K., Arjmand E., Roberson D.W. et al. (2011) Variation in surgical time-out and site marking within pediatric otolaryngology. Arch Otolaryngol Head Neck Surg. 137, 69-73

19 Shah R.K., Nussenbaum B., Kienstra M. et al. (2010) Wrong-site sinus surgery in otolaryngology. Otolaryngol Head Neck Surg. 143, 37-41

20 Haynes A.B., Weiser T.G., Berry W.R. et al. (2009) A surgical safety checklist to reduce morbidity and mortality in a global population. The New England journal of medicine. 360, 491-499 